\title{
Environment Induced Shape Change of Palladium Nanocrystals
}

\author{
Aram Yoon ${ }^{1,2}$ and Jian-Min Zuo ${ }^{1,2}$ \\ 1. Department of Materials Science \& Engineering, University of Illinois at Urbana-Champaign, Urbana, \\ Illinois 61801, USA. \\ 2. Fredrick Seitz Material Research Laboratory, University of Illinois at Urbana-Champaign, Urbana, \\ Illinois 61801, USA.
}

The exceptional catalytic activity of nanocrystals has been attributed to the large fraction of surface atoms involved in solid-gas or solid-liquid reactions. Since the surface structure is directly related to the available sites, it is important to study the shape of nanocrystals or its surface change in various environment. Environmental transmission electron microscope (ETEM) provides a versatile tool for investigating nanostructures. It enable us to control temperature and gas pressure, so that the dynamic crystal shape change of nanocrystals can be directly observed at atomic scale at various thermodynamic conditions.

Here, we report an in-situ study of the shape change of the palladium nanocrystals supported on $\mathrm{TiO}_{2}$ in pure oxygen gas using an environment TEM (Hitachi-9500) equipped with a deferential pumping system and a specially designed gas-heating holder. In order to correlate the kinetics and thermodynamic of the reaction of nanocrystals, careful experimental design and control over the experimental parameters are required. These parameters include electron beam intensity, temperature, gas pressure and time. Their effects were systematically investigated. The samples were observed at two different temperatures separately: $200{ }^{\circ} \mathrm{C}$, and $400{ }^{\circ} \mathrm{C}$. The samples were subject to the pressure change from TEM vacuum pressure of $\sim 10^{-5} \mathrm{~Pa}$ to $2.0 \times 10^{-3} \mathrm{~Pa}$ by injecting pure oxygen gas through the gas nozzle. The electron current density is maintained to be lower than $3.0 \mathrm{Acm}^{-2}$ to minimize the electron beam irradiation effect.

All the variables contribute to the kinetics and thermodynamics of the reaction and determine the crystal structure and morphology. Under different gas pressure, the nanoparticles expose different facets. As shown in Figure 1, palladium nanoparticles supported on titanium oxide mainly consist of $\{100\},\{111\}$ facets with sharp edges in low oxygen gas pressure (TEM vacuum) (Figure 1a and c), however they expanded $\{110\}$ plane facets and other higher indexed planes, making crystal shapes rounded with oxygen (Figure $1 \mathrm{~b}$ and $\mathrm{d}$ ). The result agrees with the previous theory and $\mathrm{x}$-ray studies on palladium nanoparticles $[1,2]$ and similar rounding was observed in other catalyst materials such as gold [3, 4] or platinum [5]. Interestingly, particles exposed to oxygen were often observed with the shell surrounding the palladium nanocrystal. The shell is the indication of the surface oxide. Oxides decorated the edges between the $\{111\}$ plane and the $\{110\}$ plane with the shell thickness $3.7 \AA$, and the top surface of the $\{100\}$ plane with 6.1 $\AA$ of thickness (Figure 1b).

In conclusion, the Pd nanoparticles adjust its shape and form oxide shells when it is exposed to oxygen in higher temperature. The observation of this process in ETEM provides insight into the surface dynamics and the mechanism of operating catalyst.

References:

[1] F. Mittendorfer, N. Seriani, O. Dubay, and G. Kresse, Phys. Re. B 76 (2007), p. 233413

[2] P. Nolte, A. Stierle, N. Kasper, N. Y. J. Phillipp, N. Jeutter, and H. Dosch, Nano Lett. 11 (2011), p. 4697-4700. 
[3] T. Uchiyama, H. Yoshida, Y. Kuwauchi, S. Ichikawa, S. Shimada, M. Haruta, and S. Takeda, Angew. Chem. 123 (2011), p. 10339-10342.

[4] Y. Kuwauchi, H. Yoshida, T. Akita, M. Haruta, and S. Takeda, Angew. Chem. Int. Ed 51 (2012), p. 7729-7733

[5] U. Hejral, A. Vlad, P. Nolte, and A. Stierle, J. Phys. Chem. C 117 (2013), p. 19955-19966

[6] The authors acknowledge funding from the Natioanl Science Foundation, USA, and Kwanjeong Educational Foundation, Korea. The ETEM is supported by DMR 12-29454. JMZ is partially supported by DMR 14-10596, and AY is supported by the DMR 14-10596 and Kwanjeong Educational Foundation.
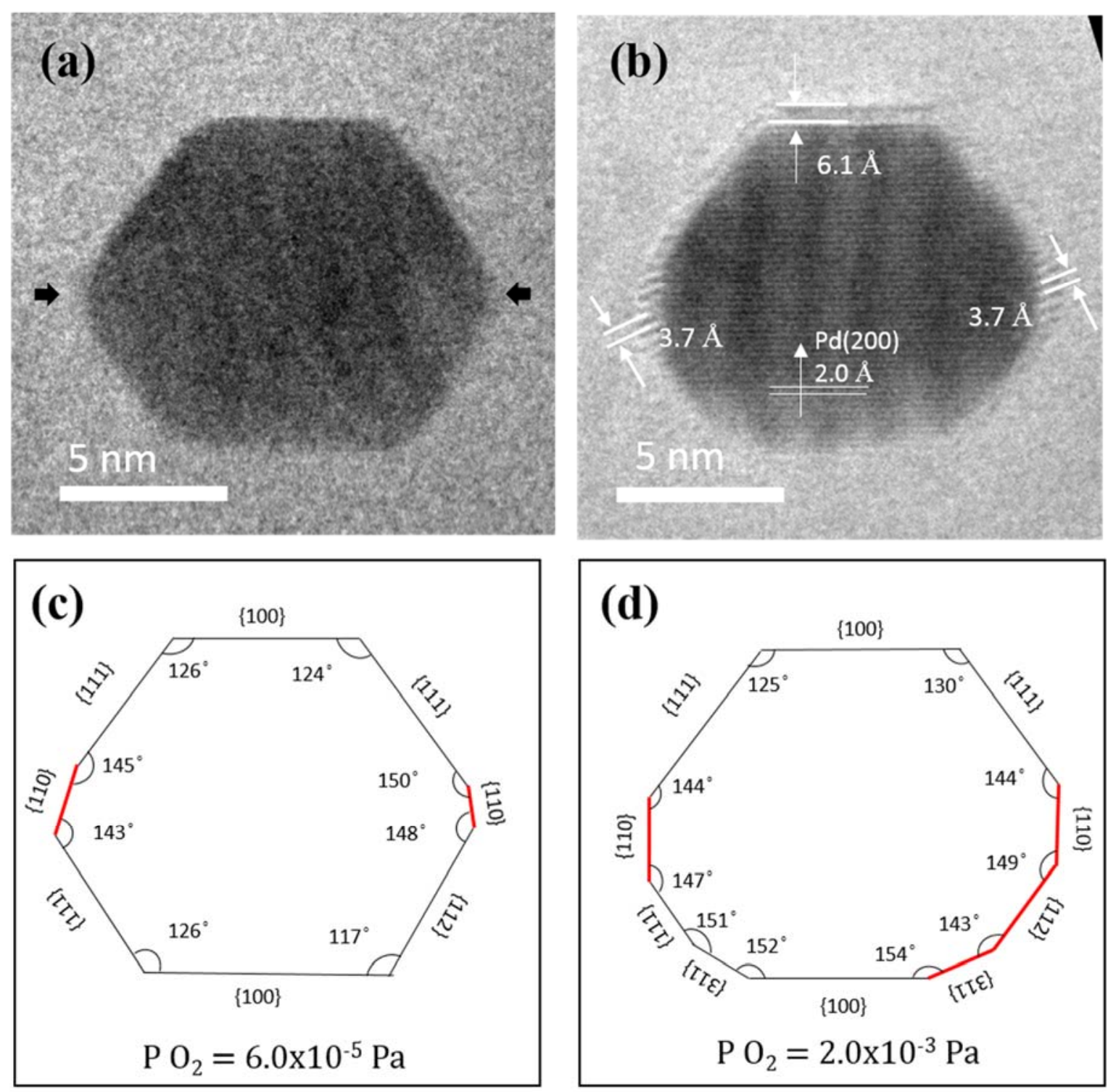

Figure 1. Palladium particle on $\mathrm{TiO}_{2}$ in (a) vacuum and (b) oxygen gas at $200{ }^{\circ} \mathrm{C}$. Two corners in (a) becomes flat in (b) resulting in a blunt shape in oxygen gas. Figure (c) and (d) are the projected polyhedron shapes of the Pd nanocrystals corresponding to (a) and (b) respectively. 